\title{
Isokinetic strength profile of subjects with proximal patellar tendinopathy
}

\author{
J.F. Kaux ${ }^{1,2}$, J.L. Croisier ${ }^{1,2}$, V, Libertiaux ${ }^{3}$ \\ 1 Physical Medicine and Sports Traumatology Department, SportS², FIFA Medical Centre of Excellence, IOC \\ Research Centre for Prevention of Injury and Protection of Athlete Health and FIMS Collaborating Centre of \\ Sports Medicine University and University Hospital of Liège, Liège, Belgium \\ 2 Department of Sport and Rehabilitation Science, University of Liège, Liège, Belgium \\ 3 Department of Clinical Sciences, Faculty of Veterinary Medicine, University of Liège, Liège, Belgium
}

\section{CORRESPONDING AUTHOR:}

Jean-François Kaux

Physical Medicine and Sports

Traumatology Department, SportS2, FIFA Medical Centre of Excellence, IOC Research Centre for Prevention of Injury and Protection of Athlete Health and FIMS Collaborating Centre of Sports Medicine University and University Hospital of Liège, Liège, Belgium,

E-mail: jfkaux@chuliege.be,

tel: +3243668241

fax: +3243667230

DOI:

10.32098/mltj.02.2019.08

LEVELOF EVIDENCE:

$2 b$

\begin{abstract}
SUMMARY
Background. Proximal patellar tendinopathy is relatively common among sportsmen. However, the strength profile of subjects with proximal patellar tendinopathies is poorly described. Purpose. We aimed to determine the isokinetic strength profile in order to estimate the difference of muscular performance between the healthy and pathological limbs of subjects suffering of this chronic pathology. Study design. cohort study. Methods. 43 subjects with chronic proximal patellar tendinopathy were involved. It has been based on the evaluation of the quadriceps and the hamstrings muscular performance of the healthy and pathological member on isokinetic dynamometer at the speed of $60 \% \mathrm{~s}$ (C60) and of $240 \% \mathrm{~s}(\mathrm{C} 240)$ in concentric mode and at the speed of $30 \% \mathrm{~s}$ (E30) in eccentric mode. A visual analogic scale of pain (VAS) has been also realized after each isokinetic test. Results. The results of the isokinetic tests comparing the healthy to the pathological limb are meaningful for the different conditions of contraction and test speeds, just like the results of the VAS associated to those tests $(\mathrm{p}<0,01)$. Indeed, pathological knees had a maximum peak torque for the quadriceps in C60 lower than healthy. Conclusion. In our study, the isokinetic results show a significant difference in performance isokinetic between the healthy and the pathological limb as well as VAS associated with these tests. However, the diversity of outcomes recorded in the patients suggests us that an individualized treatment is the case of patellar tendon pathology. Finally, it would seem that an isokinetic test in eccentric in some patients is, in addition to a test of provocation of the tendon, a pain assessment tool.
\end{abstract}

\section{KEY WORDS}

patellar tendinopathy; isokinetics; concentric; eccentric

\section{INTRODUCTION}

Patellar tendinopathy (PT) is relatively common among sportsmen $(3,7,9)$. Its incidence is reported to be in the range of $7-40 \%$ (14). Proximal patellar tendinopathies, also called jumper's knee (JK) represent two thirds of all overuse pathologies of the knee induced by volleyball or basketball practice (14). Nearly one volleyball player out of 2 will suffer from this tendon condition during his sport career. Even if there exist lots of study to understand its physiopathology, to evaluated its mechanical repercussions and to propose treatments $(1,4,10-11,17,19-23)$, however, the quadriceps strength profile of subjects with proximal patellar tendinopathies remains poorly described.
Indeed, due to the knee pain among subjects with PT, the quadriceps could be deconditioned and its strength could decrease. Moreover, this decrease of strength could persist after the decrease or disappearance of patellar tendon pain. So, a specific strengthening of the quadriceps could be useful before return to play for some patients who have to be identified.

Thus we aimed to determine the strength profile (difference of muscular performance between the healthy and pathological limb measure by an isokinetic dynamometer) of subjects suffering of this chronic pathology. This could perhaps provide the therapist with rationale information contributing to adapt their treatment for patient in such condition. 


\section{MATERIALS AND METHODS}

This protocol of our cohort study was reviewed and accepted by the Ethic Committee of the University of Liège.

\section{Patients}

43 patients (42 males, age $=29.1$ y.o. $+/-9.9$ y.o., weight $=$ $78.6 \mathrm{~kg}+/-11.8 \mathrm{~kg}$, height $=1.79 \mathrm{~m}+/-0.07 \mathrm{~m})$, all leisure sportsmen and suffering from chronic (more than 3 months) proximal PT were recruited in the Physical and Rehabilitation Department of the University Hospital of Liège. To be eligible, the patients must not have suffered from any other traumatic or micro-traumatic injury than the proximal PT on the pathologic limb, including

- muscular lesions (quadriceps, hamstrings, sural triceps), ligamentar lesions ( ACL, ILL and ELL), meniscal and tendineous.

- chondropathy and pattelar instability

- knee surgery

- any other tendinopathy of the knee extensor.

An informed consent form explaining the different modalities of the study was read and approved by the patients.

The PT was confirmed by a clinical examination (and VISA-P [12]) and a Doppler ultrasonography. The patients were then evaluated with isokinetic protocols and a pain assessment was performed by mean of a visual analog scale (VAS).

\section{Physical examination and ultrasonography}

After a thorough anamnesis, the clinician performed a physical examination. The tendon's damage was assessed by ultrasound (US) examination with a 8-15 MHz probe (Logiq S7, GE, Connecticut, USA) performed by the same investigator. The following pieces of information were recorded: echo-texture, tendon maximal thickness, ratio between the thickness of the pathological and the healthy parts of the tendon, axial and sagittal hypo-echoic length and amount of neovascularization using colour Doppler.

\section{Isokinetic testing}

Isokinetic measurements of the quadriceps and hamstrings were conducted using the Cybex Norm dynamometer (Lumex, NY, USA). Before the tests, the patients warmed up by pedaling on a stationary bike during 10 minutes at a light intensity (50 - 100 Watts).

The subject was asked to sit on the dynamometer seat with his back reclined at $85^{\circ}$. The subject was stabilized using thigh, pelvic and shoulder straps. In particular, the thigh strap prevented simultaneous hip and knee flexion during testing of the hamstring. The resistance pad was adjusted to face a point $3 \mathrm{~cm}$ above the malleoli. Before the actual test begun, the subject performed three series of 5 progressively intensified submaximal contractions. Both the pathological and the control legs were tested according to the following modalities:

- Quadriceps - 3 concentric repetitions at 60\% (QC60)

- Quadriceps -5 concentric repetitions at $240 \%$ s (QC240)

- Quadriceps - 3 eccentric repetitions at 30\% (QE30)

- Hamstring -3 concentric repetitions at $60 \% \mathrm{~s}$ (HC60)

- Hamstring -5 concentric repetitions at $240 \%$ s (HC240)

For all tests, the angular amplitude was fixed to $100^{\circ}$. Before each of these series, the subject was asked to perform 3 sub-maximal repetitions at the prescribed speed. The peak torque (PT) and the bodyweight normalized PT (PT/kg) were recorded and a VAS test was performed after each modalities for the quadriceps. The quadriceps was also tested in eccentric at $30 \%$ s. This mode of contraction and angular velocity were selected for the following reasons: 1) because eccentric allows to develop a higher strength in comparison with concentric mode, testing in eccentric should induce an increased mechanical stress in the tendon; this could be of interest in order to assess the patient's pain 2 ) in eccentric, it is easier to familiarize with a low velocity in comparison with high velocities. For this reason, low velocity warrants more accurate and safe measurements.

\section{Statistical analysis}

For each test, we ensured the data followed a normal distribution through a Shapiro-Wilk test. We then performed paired t-tests to determine if a significant difference existed between the PT (resp. PT/kg) values of the healthy and pathological limb. We also checked for correlation between the difference in PT (resp. PT $/ \mathrm{kg}$ ) values between the healthy and pathological limb and the demographic variables, the US variables and the inter-limb pain difference as expressed by the VAS.

\section{RESULTS}

A. In this section are presented the synthetic results for each of the afore mentioned analyses for all the 43 patients with chronic patellar tendinopathy.

\section{Ultrasonography}

The mean anteroposterior thickness of the pathological tendon is $8.1 \mathrm{~mm}(+/-2.1 \mathrm{~mm})$ and the associated pathological thickness to healthy thickness ratio is $1.76(+/-0.24$ 
$\mathrm{mm})$. The axial and sagittal hypo-echoic length are $6.8 \mathrm{~mm}$ +/- $6.1 \mathrm{~mm}$ and $19.0 \mathrm{~mm}+/-7.4 \mathrm{~mm}$ respectively.

\section{Isokinetic protocol}

Table 1 shows the mean and standard deviation MT values obtained for each protocol. The MTm results are presented as whisker plots in Figure $\mathbf{1}$ and Figure 2. Looking at the values in Table 1, one can see that albeit the values for the E30 tests are fairly similar those for the C60, the difference between the healthy and the pathologic limb is not significant. This observation is to be attributed to the inter-individual variability (Figure 3).

\section{Visual analog scale}

We report in Table 2 the mean values and standard deviation for the difference in VAS value between the two legs. The difference between the results at E30 are significantly higher than for the other two protocols. The eccentric loading induces a higher mechanical stress on the tendons, hence resulting in a stronger pain in the pathologic limb.

B. From this section to the end of the report, we adopt the convention that « $\Delta$ variable » stands for the arithmetic difference between the value of « variable » for the pathologic limb and the healthy limb.

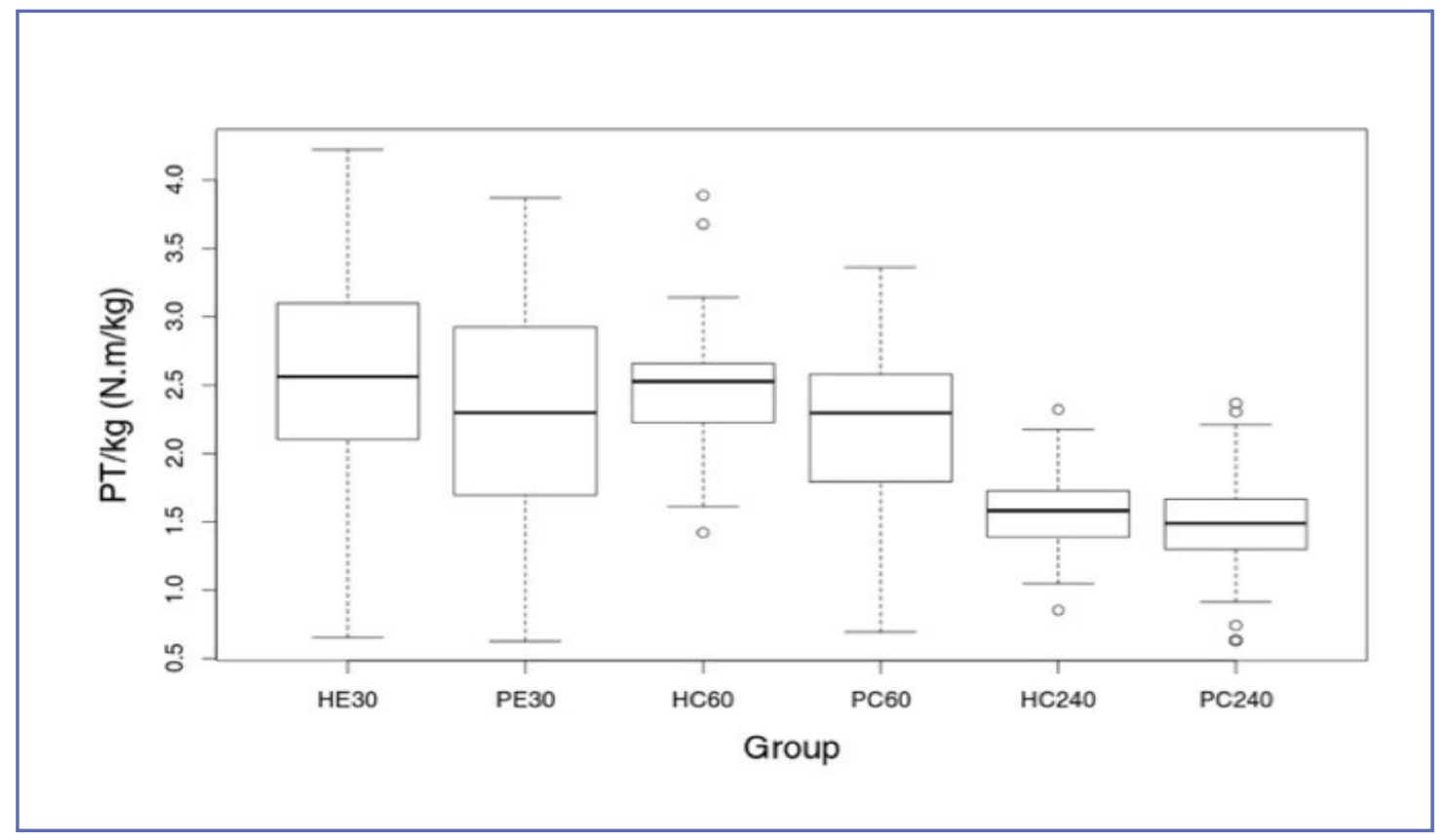

Figure 1: Bodyweight normalized peak torque whisker plot for the quadriceps. $P=P a t h o l o g i c, H=H e a l t h y$, $\mathrm{E}=$ Eccentric, $\mathrm{C}=$ Concentric

Table I. Maximum torque mean values and standard deviations for each isokinetic protocol. (*) indicates a significant difference between the healthy and the pathologic limb.

\begin{tabular}{lllll}
\hline & & E30 & C60 & C240 \\
\hline Quadriceps & Healthy (N.m) & $199.6 \pm 72.0$ & $193.4 \pm 35.6$ & $123.2 \pm 27.4$ \\
\hline Hamstrings & Pathologic (N.m) & $174.8 \pm 67.3$ & $170.5 \pm 44.6(*)$ & $114.3 \pm 30.0$ \\
\hline & Healthy (N.m) & & $103.6 \pm 22.8$ & $66.0 \pm 15.0$ \\
\hline
\end{tabular}




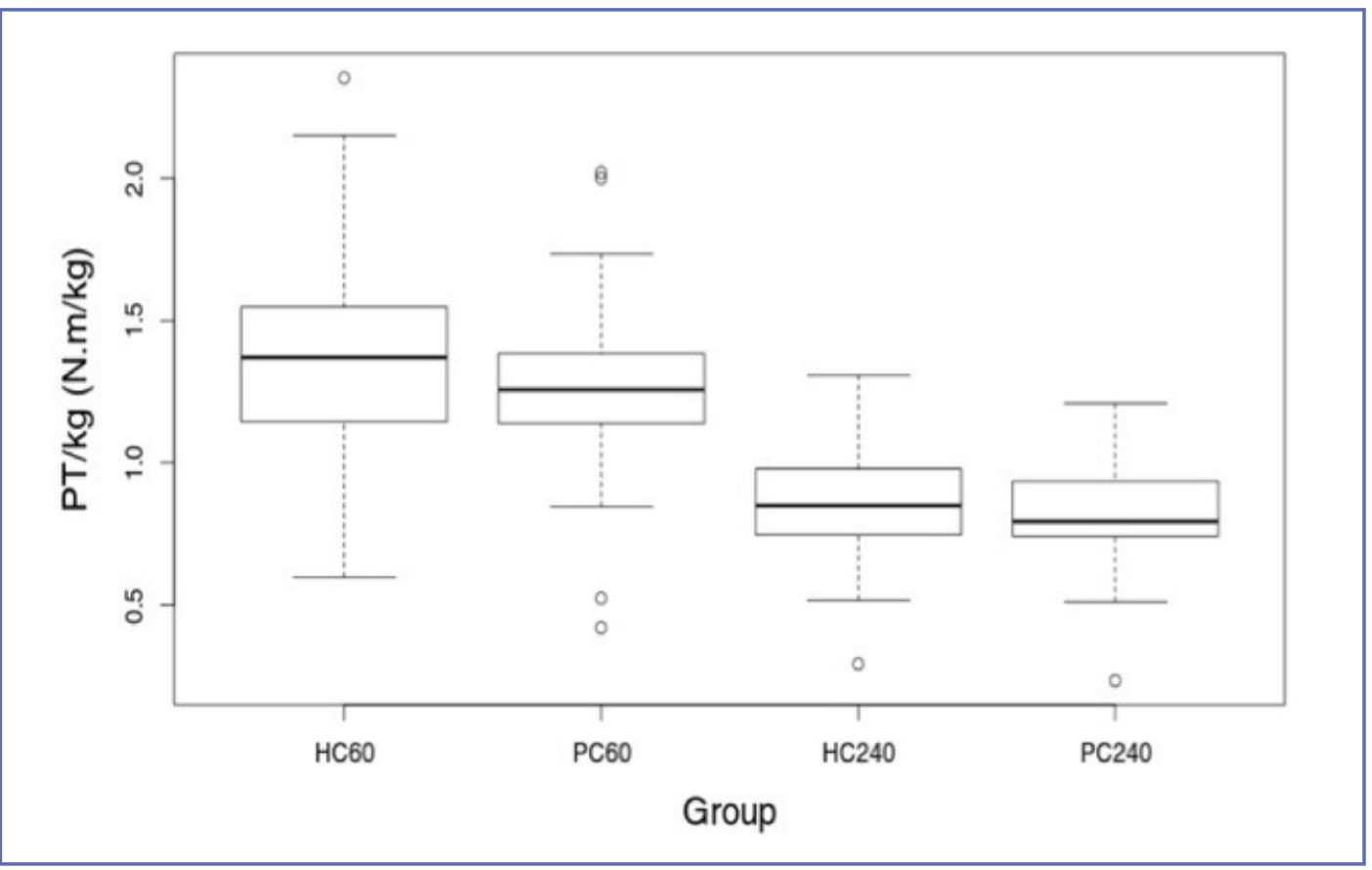

Figure 2: Bodyweight normalized peak torque whisker plot for the hamstrings. P=Pathologic, $\mathrm{H}=$ Healthy, $\mathrm{E}=$ Eccentric, $\mathrm{C}=$ Concentric

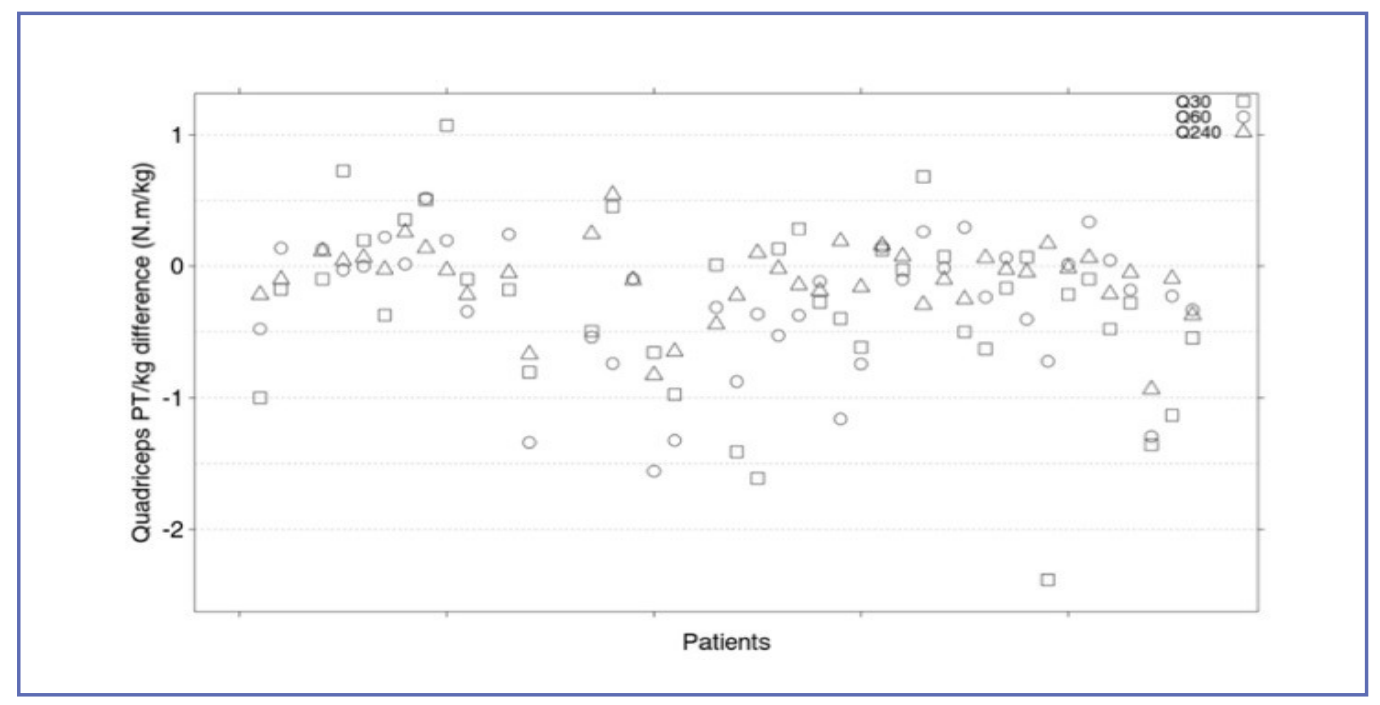

Figure 3: Bodyweight normalized peak torque difference scatter plot for the quadriceps

Table II: Pain difference between the pathologic and the contralateral limb assessed by a visual analog scale

\begin{tabular}{ccc}
\hline$\Delta$ VAS QE30 & $\Delta$ VAS QC60 & $\Delta$ VAS QC240 \\
\hline $5.08+/-3.02$ & $3.43 \pm 2.80$ & $2.84 \pm 2.35$ \\
\hline
\end{tabular}




\section{Demographic variables}

Statistically significant correlation was found between the patients' height and the hamstrings $\triangle \mathrm{PT} / \mathrm{kg}$ at both $\mathrm{C} 60 \mathrm{(r}$ $=0.38, \mathrm{p}=0.014)$ and C240 $(\mathrm{r}=0.3, \mathrm{p}=0.05)$. No other correlation was found involving demographic variables.

\section{US variables}

Although it is generally admitted that there seems to be no correlation between muscle strength pain, and imagery, we found that the $\Delta \mathrm{PT} / \mathrm{kg}$ for the QE30 was negatively correlated with the patellar tendon axial hypo-echoic zone length $(\mathrm{r}=-0.4, \mathrm{p}=0.009)$ and with the anteroposterior dimension $(\mathrm{r}=-0.36, \mathrm{p}=0.02)$. We also found a positive correlation between the pathologic to healthy tendon thickness ratio and $\triangle \mathrm{VAS}$ for the quadriceps in $\mathrm{E} 30(\mathrm{r}=0.32, \mathrm{p}=$ $0.05)$ and in $\mathrm{C} 60(\mathrm{r}=0.32, \mathrm{p}=0.04)$.

\section{Peak torque and bodyweight normalized peak torque}

Only the PT $/ \mathrm{kg}$ for the quadriceps $(\mathrm{p}=0.03)$ and the hamstrings $(\mathrm{p}=0.04)$ in $\mathrm{C} 60$ were significantly different between the two legs, and this despite the relatively important inter-individual variability. Indeed, there were as many (12) patients with a $25 \%$ and more relative difference in peak torque per mass unit as there were with a $10 \%$ and less for the $\mathrm{C} 60$ test.

\section{Visual analog scale}

The $\triangle$ VAS were all significantly different from 0 , with $\mathrm{p}$ values inferior to $5 \mathrm{e}-9$. A correlation was found between $\triangle \mathrm{PT} / \mathrm{kg}$ QC60 and the corresponding VAS $(\mathrm{r}=-0.36, \mathrm{p}=$ $0.026)$.

\section{DISCUSSION}

The physiopathology and the tretaments of tendinopathies, and especially of the patellar tendinopathy, are more and more studied but remain not clearly understood $(1,4,13,16$ 17,19-23). Moreover, the repercussion of this condition on strength of the thigh is poorly described. Thus in our original series, we aimed to objective the precise isokinetic concentric and eccentric strength profile of subjects with JK. These observations could help physicians to take care and improve the treatment of these patients. Indeed, the latter generally aims to clearing the symptoms and restoring the tendon's structure and ability to bear loads. However, few to no study has been devoted to the investigation of the loss of muscular strength in the pathological limb $(1,4,17,19-23)$. Thus, the main objective of this report was to quantify this parameter and look for a trend in the muscle weakness and muscular unbalance between the two legs after recovery.

The important scatter in the isokinetic protocol (Table 1, Figure 1 and Figure 2) results clearly demonstrate the non existence of a consistent muscle strength PT $(/ \mathrm{kg})$-based profile at different loading velocities, and underlines the importance for the clinicians to apply a personalized treatment to each patient in order to recover his maximum force. It is therefore relevant, in the case of a patellar tendinopathy, to assess the quadriceps strength (with an isokinetic dynamometer) at the end of the prescribed treatment to evaluate the recovery (15).

The relationship between the $\Delta \mathrm{PT} / \mathrm{kg}$ for the quadriceps tested in eccentric mode and the measurements made on the ultrasound images is of greater interested as it relates together an objective, functional measurement of the patient's force deficit with an objective, anatomical measurement of the amount of damage to the tendon.

It is generally admitted that the pain felt by the patient caused by its tendinopathy is not necessarily reflective of the tendon's damage $(2,4,5,18)$. However, in our study, the pain assessment was realized after an isokinetic protocol that caused mechanical stress on the tendon in a repeatable manner. Therefore, the fact that we found a correlation between the $\triangle \mathrm{VAS}$ for E30 and the pathologic to healthy tendon thickness ratio does not seem contradictory to the accepted observation that the tendon's appearance on ultrasound images is generally not a good indicator of the intrinsic pain felt by the patient.

Our results also suggest that the isokinetic test QC60 is the one that can emphasize the force deficit caused by the tendinopathy on the pathologic limb and could be an indicator of good evolution after treatement (10), while the E30 caused the most intense pain. A force deficit is an indicator that the treatment must be continued to achieve a full recovery.

Finally, according to the VAS results, the isokinetic eccentric testing of the quadriceps induces a significantly more intense pain than the concentric protocols (6). Its physiopathologic mechanism is also the same than the one that caused the injury $(1,22)$. Therefore, it can be used to create a mechanical stress on the tendon for a reliable pain assessment.

By comparing the pain before the first session of physical therapy and at the end of it in this repeatable manner, the effect of the treatment can be objectively quantified.

However, even though the inter-limb difference in PT in the E30 protocol does not possess a prognostic value, one can wonder about the tendon's capacity to bear the sport-induced mechanical loads it is found to be not negligible $(1,22)$. On the other hand, this very test could cause a secondary injury as it is likely to be more demanding on the musculotendinous system than the sport activity. Clinicians 
should therefore balance the pros and cons of this particular protocol.

In our study, it was not possible to obtain the PT values on both legs since the patients were tested with an existing injury. It would be interesting to design a new study for which the participants would be followed regularly until full recovery is assessed.

\section{CONCLUSION}

In this study, we investigated a muscle strength loss profile in patients suffering from proximal pattelar tendinopathy. Amongst the five isokinetic protocols used, we found out that the quadriceps concentric contraction at $60 \mathrm{deg} / \mathrm{s}$ was the only one that consistently displayed a significant strength difference between the pathologic and the contralateral limb.

Our observations suggest that the treatment of subjects with JK should be individualized and that an isokinetic test should be carried out at the end of rehabilitation program. This way, the therapist will evaluate the individual strength profile of his patient and adapt his treatment in consequences, possibly decreasing the risk of other injury or re-injury.

Sub-maximal eccentric programs are frequently used for treating tendinopathies. Yet, according to our best knowledge, the use of maximal eccentric contractions in jumper's knee testing is poorly described. Its antagonistic function might induce the pain felt in the tendons. Furthermore, it is well established that the force developed in eccentric loading is higher than its concentric counterpart and implicitly creates more mechanical stress on the musculotendinous structures. It should therefore be a good indicator of muscle strength deficit in the pathologic limb but also to induce a similar pain to that which can be felt under competitive conditions. This assessment could be included in the return to play tests after JK.

Finally, the QE30 protocol seems very indicated to induce repeatable mechanical stress on the tendon to consistently evalutate the pain experienced by the patients.

\section{Conflict of Interest}

The authors declare that they have no conflict of interest (24)

\section{REFERENCES}

1. Bisseling RW, Hof AL, Bredeweg SW, Zwerver J, Mulder T. Are the take-off and landing phase dynamics of the volleyball spike jump related to patellar tendinopathy? Br J Sports Med, 2008. 42(6): p. 483-9.
2. Cook JL, Khan KM, Kiss ZS, Coleman BD, Griffiths L. Asymptomatic hypoechoic regions on patellar tendon ultrasound: A 4-year clinical and ultrasound followup of 46 tendons. Scand J Med Sci Sports, 2001. 11(6): p. 321-7.

3. Durcan L, Coole A, McCarthy E, Johnston C, Webb MJ, O'Shea FD, Gissane C, Wilson F. The prevalence of patellar tendinopathy in elite academy rugby: a clinical and imaging study. J Sci Med Sport, 2014. 17(2): p. 173-6

4. Edwards S, Steele JR, McGhee DE, Purdam CR, Cook JL. Asymptomatic players with a patellar tendon abnormality do not adapt their landing mechanics when fatigued. J Sports Sci, 2017. 35(8): p. 769-776.

5. Giacchino M, Caresio C, Gorji NE, Molinari F, Massazza G, Minetto MA. Quantitative analysis of patellar tendon size and structure in asymptomatic professional players: sonographic study. Muscles Ligaments Tendons J, 2017. 7(3): p. 449-458.

6. Hody S, Leprince P, Sergeant K, Renaut J, Croisier JL, Wang F, Rogister B. Human muscle proteome modifications after acute or repeated eccentric exercises. Med Sci Sports Exerc, 2011. 43(12): p. 2281-96.

7. Janssen I, van der Worp H, Hensing S, Zwerver J. Investigating Achilles and patellar tendinopathy prevalence in elite athletics. Res Sports Med, 2018. 26(1): p. 1-12.

8. Kaux JF, Croisier JL, Bruyere O, Rodriguez De La Cruz C, Forthomme B, Brabant G, Lapraille S, Lonneux V, Noel D, Le Goff C, Gothot A, Collette J, Crielaard JM. One injection of platelet-rich plasma associated to a submaximal eccentric protocol to treat chronic jumper's knee. J Sports Med Phys Fitness, 2015. 55(9): p. 953-61

9. Kaux JF, Crielaard JM. [Tendon et tendinopathie]. Journal de Traumatologie du Sport, 2014. 31(4): p. 235-240.

10. Kaux JF, Bruyere O, Croisier JL, Forthomme B, Le Goff C, Crielaard JM. One-year follow-up of platelet-rich plasma infiltration to treat chronic upper patellar tendinopathies Acta Orthopaedica Belgica, 2015.

11. Kaux JF, Croisier JL, Forthomme B, Le Goff C, Buhler F, Savanier B, Delcour S, Gothot A, Crielaard JM. Using platelet-rich plasma to treat jumper's knees: Exploring the effect of a second closely-timed infiltration. J Sci Med Sport, 2015.

12. Kaux JF, Delvaux F, Oppong-Kyei J, Beaudart C, Buckinx F, Croisier JL, Forthomme B, Crielaard JM, Bruyère O. Cross-cultural Adaptation and Validation of the Victorian Institute of Sport Assessment-Patella Questionnaire for French-Speaking Patients With Patellar Tendinopathy. J Orthop Sports Phys Ther, 2016. 46(5): p. 384-90.

13. Kaux JF, Drion P, Libertiaux V, Colige A, Hoffmann A, Nusgens B, Besançon B, Forthomme B, Le Goff C, Franzen R, Defraigne JO, Cescotto S, Rickert M, Crielaard JM, Croisier JL. Eccentric training improves tendon biomechanical properties: a rat model. J Orthop Res, 2013. 31(1): p. 119-24.

14. Kaux JF, Forthomme B, Goff CL, Crielaard JM, Croisier JL. Current opinions on tendinopathy. J Sports Sci Med, 2011. 10(2): p. 238-53.

15. Kaux JF, Forthomme B, Namurois MH, Bauvir P, Defawe N, Delvaux F, Lehance C, Crielaard JM, Croisier JL. Description of a standardized rehabilitation program based on sub-maximal eccentric following a platelet-rich plasma infiltration for jumper's knee. Muscles Ligaments Tendons J, 2014. 4(1): p. $85-9$. 
16. Kaux JF, Libertiaux V, Leprince P, Fillet M, Denoel V, Wyss C, Lecut C, Gothot A, Le Goff C, Croisier JL, Crielaard JM, Drion P. Eccentric Training for Tendon Healing After Acute Lesion: A Rat Model. Am J Sports Med, 2017. 45(6): p. 14401446.

17. Lee WC, Zhang ZJ, Masci L, Ng GYF, Fu SN. Alterations in mechanical properties of the patellar tendon is associated with pain in athletes with patellar tendinopathy. Eur J Appl Physiol, 2017. 117(5): p. 1039-1045.

18. McAuliffe S, McCreesh K, Culloty F, Purtill H, O'Sullivan K. Can ultrasound imaging predict the development of Achilles and patellar tendinopathy? A systematic review and meta-analysis. Br J Sports Med, 2016. 50(24): p. 1516-1523.

19. Morton S, Williams S, Valle X, Diaz-Cueli D, Malliaras P, Morrissey D. Patellar Tendinopathy and Potential Risk Factors: An International Database of Cases and Controls. Clin J Sport Med, 2017. 27(5): p. 468-474.

20. Scattone Silva R, Purdam CR, Fearon AM, Spratford WA, Kenneally-Dabrowski C, Preston P, Serrão FV, Gaida JE.
Effects of Altering Trunk Position during Landings on Patellar Tendon Force and Pain. Med Sci Sports Exerc, 2017. 49(12): p. 2517-2527.

21. Torres R, Ferreira J, Silva D, Rodrigues E, Bessa IM, Ribeiro F. Impact of Patellar Tendinopathy on Knee Proprioception: A Cross-Sectional Study. Clin J Sport Med, 2017. 27(1): p. 31-36.

22. Van der Worp H, de Poel HJ, Diercks RL, van den AkkerScheek I, Zwerver J. Jumper's knee or lander's knee? A systematic review of the relation between jump biomechanics and patellar tendinopathy. Int J Sports Med, 2014. 35(8): p. 714-22.

23. Zhang ZJ, Ng GYF, Lee WC, Fu SN. Increase in passive muscle tension of the quadriceps muscle heads in jumping athletes with patellar tendinopathy. Scand J Med Sci Sports, 2017. 27(10): p. 1099-1104.

24. Padulo J., Oliva F., Frizziero A., Maffulli N. Muscles, Ligaments and Tendons Journal - Basic principles and recommendations in clinical and field Science Research: 2018 update. MLTJ 2018; 8(3): 305 - 307. 